\title{
Mean Field and the Single Homopolymer
}

\author{
S. Pasquali ${ }^{1}$, J.K. Percus ${ }^{2}$ \\ ${ }^{1}$ Laboratoire de Physico-Chime Théorique, UMR Gulliver CNRS-ESPCI 7083, \\ 10 rue Vauquelin, 75231 Paris Cedex 05, France. \\ ${ }^{2}$ Courant Institute and Physics Department NYU, 251 Mercer St. New York, NY 10012
}

(Dated: November 21, 2018)

\begin{abstract}
We develop a statistical model for a confined chain molecule based on a monomer grand canonical ensemble. The molecule is subject to an external chemical potential, a backbone interaction, and an attractive interaction between all monomers. Using a Gaussian variable formalism and a mean field approximation, we analytically derive a minimum principle from which we can obtain relevant physical quantities, such as the monomer density, and we explore the limit in which the chain is subject to a tight confinement. Through a numerical implementation of the minimization process we show how we can obtain density profiles in three dimensions for arbitraty potentials, and we test the limits of validity of the theory.
\end{abstract}

PACS numbers: 05.20.Gg, 82.35.Lr, 87.15.A-

\section{INTRODUCTION}

The classical theory of fluids in thermal equilibrium is a highly developed discipline. Along with specific physically motivated approximations have come tools of more general validity and utility. Studies of polymers have of course been extensive, and useful relationships between polymer melts and fluids established [1], as well as between single polymers and fluids. This paper represents an initial investigation, aimed at understanding which concepts borrowed from bulk fluid studies $[2,3]$ still remain relevant in a polymer setting. Our focus will be on a single polymer chain confined by external forces, and to minimize the needed information input, on single homopolymers. We will also attend in the main to idealized models in which the polymer is simply a chain of unit monomers of a few degrees of freedom, but will indicate how this restriction can be rewardingly removed, on the way to a realistic polymer representation.

We will aim at both analytic simplicity and reasonable suitability for the ultimately necessary computational procedures. For the former, we will work in a "monomer grand-ensemble" $[4,5]$ in which the number of monomers per polymer is distributed, but will show that this need not be a drawback. For the latter, we will favor minimum principles to be able to better control computations. This does restrict the category of systems to be studied e.g. to purely attractive 
pair interactions, and will be generalized in later work, soon to be reported, as such restrictions are removed.

\section{THE REFERENCE SYSTEM}

\section{A. Notation}

Let us be a bit more explicit. We have in mind an ordered chain of $N$ equivalent monomers, the $j^{\text {th }}$ being specified by its degrees of freedom $r_{j}$. The order is maintained by a symmetric next neighbor interaction potential of Boltzmann factor $w\left(r_{i}, r_{i+1}\right)$, depending of course on the inverse temperature $\beta$. Any two monomers can also interact via an interaction Boltzmann factor $e\left(r_{i}, r_{j}\right)=\exp \left(-\beta \phi\left(r_{i}, r_{j}\right)\right)$ and, crucially for the applications we have in mind, the polymer is constrained by an external potential $u\left(r_{i}\right)$.

It is now convenient to imagine that the homopolymer in question is both in thermal equilibrium and in number equilibrium, i.e. that it is the result of monomer addition and absorption from a bath of non-interacting monomers. The reaction equilibrium is analogous to that of the grand canonical ensemble for a fluid, but with a significant difference. Suppose that $Q^{(m)}$ is the monomer canonical partition function in its center of mass coordinate system, $Q_{N}^{(p)}$ of the $N$-monomer polymer $(N \geq 1$, to recognize an object as a polymer). Then if the full system contains $N^{\prime}$ monomers, $N$ of which are bound together, in a volume $V$, the full system partition function will be:

$$
Q_{N^{\prime}}^{t o t}=\sum_{N \geq 1} \frac{\left(V Q^{(m)}\right)^{N^{\prime}-N}}{\left(N^{\prime}-N\right) !} Q_{N}^{(p)} .
$$

Since the monomers in the absence of a polymer would have a partition function:

$$
Q_{N^{\prime}}^{(m)}=\frac{\left(V Q^{(m)}\right)^{N^{\prime}}}{N^{\prime} !}
$$

that attributed to the polymer will take on the form:

$$
\begin{aligned}
\Xi^{(p)}\left[\zeta_{0}\right] & =\lim _{N^{\prime} \rightarrow \infty, N^{\prime} / V \text { fixed }} \frac{N^{\prime} !}{\left(V Q^{(m)}\right)^{N^{\prime}}} Q_{N^{\prime}}^{t o t} \\
& =\sum_{N \geq 1} \zeta_{0}^{N} Q_{N}^{(p)}, \quad \text { where } \zeta_{0}=\left(N^{\prime} / V\right) / Q^{(m)} .
\end{aligned}
$$

The obvious analogy with a fluid grand partition function (with the weight $1 / N$ ! excised) will be very useful indeed.

The computation of $\Xi^{(p)}\left(\zeta_{0}\right)$, which generates all thermodynamics and expectations in a thermal ensemble, is of course too general to be explicitely solvable, except in very special circumstances. 
Let us therefore start with the evaluation of $\Xi^{(p)}\left(\zeta_{0}\right)$ for what may be regarded as the backbone of the polymer, that in which the arbitrary pair mutual interaction $\phi\left(r_{i}, r_{j}\right)$ is set equal to zero. In this case, we have at once:

$$
\begin{aligned}
\zeta_{0}^{N} Q_{N}^{(p)}= & \int \zeta\left(r_{1}\right) w\left(r_{1}, r_{2}\right) \zeta\left(r_{2}\right) \ldots \zeta\left(r_{N-1}\right) w\left(r_{N-1}, r_{N}\right) \zeta\left(r_{N}\right) d r^{N} \\
\text { where } \quad & \zeta(r)=\zeta_{0} e^{-\beta u(r)}=e^{\beta \mu(r)}
\end{aligned}
$$

or regarding $w\left(r, r^{\prime}\right)$ as the kernel of an integral operator $\mathrm{w}, \zeta(r) \delta\left(r-r^{\prime}\right)$ as that of the diagonal operator $\zeta$, and the symbol $|1\rangle$ denoting the vector whose components are 1 ,

$$
\Xi^{(p)}[\zeta]=\left\langle 1\left|\left(\zeta^{-1}-w\right)^{-1}\right| 1\right\rangle
$$

subject of course to convergence of the series (4). The corresponding "grand potential" is:

$$
\Omega^{(p)}[\zeta]=-\frac{1}{\beta} \ln \left\langle 1\left|\left(\zeta^{-1}-w\right)^{-1}\right| 1\right\rangle
$$

and as an immediate consequence the monomer density is given by

$$
\begin{aligned}
n(r) & =-\beta \zeta(r) \frac{\delta}{\delta \zeta(r)} \Omega^{(p)}[\zeta] \\
& =\left\langle 1\left|\left(\zeta^{-1}-w\right)^{-1}\right| r\right\rangle\left\langle r\left|\left(\zeta^{-1}-w\right)^{-1}\right| 1\right\rangle \zeta^{-1}(r) /\left\langle 1\left|\left(\zeta^{-1}-w\right)^{-1}\right| 1\right\rangle
\end{aligned}
$$

\section{B. Number Distribution}

The absence of the statistical weight $1 / N$ ! suggests an unusual distribution of monomer number. To verify this, we first observe from (9) that if $\hat{N}$ denotes the monomer number in a given configuration, than

$$
\begin{aligned}
N=\langle\hat{N}\rangle & =\int n(r) d r=\left\langle 1\left|\left(\zeta^{-1}-w\right)^{-1} \zeta^{-1}\left(\zeta^{-1}-w\right)^{-1}\right| 1\right\rangle / \Xi^{(p)} \\
& =\left\langle\zeta^{1 / 2}\left|\left(I-\zeta^{1 / 2} w \zeta^{1 / 2}\right)^{-2}\right| \zeta^{1 / 2}\right\rangle /\left\langle\zeta^{1 / 2}\left|\left(I-\zeta^{1 / 2} w \zeta^{1 / 2}\right)^{-1}\right| \zeta^{1 / 2}\right\rangle \\
& =1 /\left(1-\lambda_{0}\right)+\mathcal{O}\left(1 /\left(1-\lambda_{1}\right)\right)
\end{aligned}
$$

where $\lambda_{0}=\lambda_{\max }\left(\zeta^{1 / 2} w \zeta^{1 / 2}\right)$ and $\lambda_{1}$ is the next largest eigenvalue; $\lambda_{1}<\lambda_{0}$ in a confined system. If $N$ is large, then

$$
\Xi^{(p)}(\zeta)=\left\langle\zeta^{1 / 2}\left|\left(I-\zeta^{1 / 2} w \zeta^{1 / 2}\right)^{-1}\right| \zeta^{1 / 2}\right\rangle
$$

is dominated by the "resonance" at $\lambda_{0}$, so that if

$$
\left(\zeta^{1 / 2} w \zeta^{1 / 2}\right) \psi_{\lambda_{0}}=\lambda_{0} \psi_{\lambda_{0}}
$$


with normalized $\psi$, then

$$
\Xi^{(p)}[\zeta] \sim \frac{1}{1-\lambda_{0}}\left\langle\psi_{\lambda_{0}}\left|\zeta^{1 / 2}\right| 1\right\rangle^{2}
$$

There are now two consequences. On the one hand, we have

$$
\begin{aligned}
\left\langle e^{i \theta \hat{N}}\right\rangle & =e^{i \theta \zeta_{0} \partial / \partial \zeta_{0}} \Xi^{(p)}[\zeta] / \Xi^{(p)}[\zeta] \\
& =\Xi^{(p)}\left[\zeta e^{i \theta}\right] / \Xi^{(p)}[\zeta] \\
& \cong\left(1-\lambda_{0}\right) /\left(1-\lambda_{0} e^{i \theta}\right)
\end{aligned}
$$

so that the distribution of $\hat{N}$ is given by

$$
f(N)=\operatorname{coef} e^{i N \theta}\left(1-\lambda_{0}\right) /\left(1-\lambda_{0} e^{i \theta}\right)=\lambda_{0}^{N}\left(1-\lambda_{0}\right)
$$

the very broad geometric distribution. It would appear that the $N$-ensemble must give a very poor representation of a given $N$. But on the other hand, we have from (5)

$$
\begin{aligned}
Q_{N} & =\zeta_{0}^{-N}\left\langle\zeta^{1 / 2}\left|\left(\zeta^{1 / 2} w \zeta^{1 / 2}\right)^{N-1}\right| \zeta^{1 / 2}\right\rangle \\
& \cong \zeta_{0}^{-N} \lambda_{0}^{N-1}\left\langle\psi_{\lambda_{0}} \mid \zeta^{1 / 2}\right\rangle^{2},
\end{aligned}
$$

so that if $F_{N}$ is the canonical Helmholtz free energy,

$$
\begin{aligned}
\beta F_{N}-\beta \Omega^{(p)} & =-\ln Q_{N}+\ln \Xi^{(p)}[\zeta] \\
& \cong N \ln \zeta_{0}-(N-1) \ln \lambda_{0}-\ln \left(1-\lambda_{0}\right)
\end{aligned}
$$

It follows that for any ( $N$-independent) parameter variation

$$
\beta\left(\delta F_{N}-\delta \Omega^{(p)}\right)=\left(\frac{1}{1-\lambda_{0}}-\frac{N-1}{\lambda_{0}}\right) \delta \lambda
$$

which vanishes if $\zeta_{0}$ in (14) is chosen so that

$$
N=1 /\left(1-\lambda_{0}\right)
$$

We conclude that for this choice of $\lambda_{0}$, expectations at fixed $N$ and in the monomer number ensemble are in fact identical to leading order.

\section{Minimum Principle}

With the confidence that the large spread of monomer number does not detract from the usefulness of $\Omega^{(p)}$, we proceed. The original form (6) is simplest to use for the construction of an 
associated minimum principle. It depends upon the fact if $K$ is positive semi-definite, and $a$ and $\psi$ (not to be confused with $\psi_{\lambda}$, which we will not need again until later) are arbitrary, then according to the Schwartz inequality, we have

$$
\begin{aligned}
\left\langle a\left|K^{-1}\right| a\right\rangle\langle\psi|K| \psi\rangle & =\left\langle K^{-1 / 2} a \mid K^{-1 / 2} a\right\rangle\left\langle K^{1 / 2} \psi \mid K^{1 / 2} \psi\right\rangle \\
& \geq\left\langle K^{-1 / 2} a \mid K^{1 / 2} \psi\right\rangle^{2}=\langle a \mid \psi\rangle^{2}
\end{aligned}
$$

so that $\left.\left\langle a\left|K^{-1}\right| a\right\rangle \geq\langle a \mid \psi\rangle^{2} /<\psi|K| \psi\right\rangle$. Then indeed

$$
\operatorname{Max}_{\psi} \frac{\langle a \mid \psi\rangle^{2}}{\langle\psi|K| \psi\rangle}=\left\langle a\left|K^{-1}\right| a\right\rangle \quad \text { at } K \psi=\frac{\langle\psi|K| \psi\rangle}{\langle a \mid \psi\rangle} a,
$$

and in the context of (6), we conclude that

$$
\beta \Omega^{(p)}[\zeta]=\operatorname{Min}_{\psi}\left[\ln \left\langle\psi\left|\zeta^{-1}-w\right| \psi\right\rangle-2 \ln \langle 1 \mid \psi\rangle\right]
$$

\section{THE MEAN FIELD STRATEGY}

Our task is now to take into account the mutual interaction $\phi_{1}\left(r_{i}, r_{j}\right)$ which while typically fairly short range in space, can be very long range along the polymer chain. We will confine our attention to purely attractive (negative definite) potentials, and to avoid confusion will set

$$
\phi_{1}\left(r_{i}, r_{j}\right)=-\phi\left(r_{i}, r_{j}\right)
$$

where $\phi$ is positive definite (as a continuous matrix). The assumption of pure attraction distorts local properties of the polymer, but permits large scale effects such as globularity to proceed unhindered.

At first, we need not restrict our attention to polymer chains. Quite generally, if $W_{0}^{(N)}\left(r^{N}, \mu\right)$ is the suitably weighted Boltzmann factor for the $N$-unit configuration in the absence of $\phi$, but with local chemical potential $\mu(r)$, the partition function for the interacting system can be written as

$$
\begin{aligned}
\Xi[\mu, \phi] & =\sum_{N} \int \ldots \int W_{0}^{(N)}\left(r^{N}, \mu\right) e^{\frac{\beta}{2} \sum_{i, j}^{\prime} \phi\left(r_{i}, r_{j}\right)} d r^{N} \\
& =\sum_{N} \int \ldots \int W_{0}^{(N)}\left(r^{N}, \mu\right) e^{-\frac{\beta}{2} \sum_{i} \phi_{D}\left(r_{i}\right)} e^{\frac{\beta}{2} \sum_{i, j} \phi\left(r_{i}, r_{j}\right)} d r^{N} \\
& =\sum_{N} \int \cdots \int W_{0}^{(N)}\left(r^{N}, \mu\right) e^{-\frac{\beta}{2} \sum_{i} \phi_{D}\left(r_{i}\right)} e^{\frac{\beta}{2} \iint \hat{n}(r) \phi\left(r, r^{\prime}\right) \hat{n}\left(r^{\prime}\right) d r d r^{\prime}} d r^{N}
\end{aligned}
$$

where $\sum^{\prime}$ omits the $i=j$ contribution, $\phi_{D}$ is the diagonal part of the matrix $\phi\left(r_{i}, r_{j}\right)$, and

$$
\hat{n}(r)=\sum_{i} \delta\left(r-r_{i}\right)
$$


is the "microscopic" density. The device of Kac, Siegert, Hubbard and Stratonovich [6-9] is to represent the Gaussian in (31) (in obvious notation) as a functional Laplace transform

$$
\begin{aligned}
e^{\frac{\beta}{2} \hat{n} \cdot \phi \hat{n}} & =\int e^{-\frac{\beta}{2} v \cdot \phi^{-1} v} e^{-\beta v \cdot \hat{n}} D v / \int e^{-\frac{\beta}{2} v \cdot \phi^{-1} v} D v \\
& =\int e^{-\beta \sum_{i} v\left(r_{i}\right)} e^{-\frac{\beta}{2} v \cdot \phi^{-1} v} D v / \int e^{-\frac{\beta}{2} v \cdot \phi^{-1} v} D v
\end{aligned}
$$

Since $\sum_{N} \int \ldots \int W_{0}^{(N)}\left(r^{N}, \mu\right) d r^{N}=\Xi_{0}[\mu]$, eq.(31) can thereby be rewritten as

$$
\Xi[\mu, \phi]=\int \Xi_{0}\left[\mu-\frac{1}{2} \phi_{D}-v\right] e^{-\frac{\beta}{2} v \cdot \phi^{-1} v} D v / \int e^{-\frac{\beta}{2} v \cdot \phi^{-1} v} D v
$$

with a possible interpretation that the interaction $-\phi$ has been replaced by an ensemble average over a fluctuating external field $v(r)$, serving as a sort of graviton shutting back and forth between units.

The kernel of (37) is a Boltzmann factor in field space, and so we may define a field average as

$$
\langle\langle G[v]\rangle\rangle=\int G[v] \Xi_{0}\left[\mu-\frac{1}{2} \phi_{D}-v\right] e^{-\frac{\beta}{2} v \cdot \phi^{-1} v} D v / \Xi[\mu, \phi]
$$

A very suggestive consequence of this notation follows from the observation that for the density $n(r)$,

$$
\begin{aligned}
n(r) & =\frac{1}{\Xi[\mu, \phi]} \frac{\delta}{\delta \beta \mu(r)} \Xi[\mu, \phi] \\
& =-\int \frac{\delta}{\delta \beta \mu(r)} \Xi_{0}\left[\mu-\frac{1}{2} \phi_{D}-v\right] e^{-\frac{\beta}{2} v \cdot \phi^{-1} v} D v / \Xi[\mu, \phi] \int e^{-\frac{\beta}{2} v \cdot \phi^{-1} v} D v,
\end{aligned}
$$

or integrating by parts in v-space (assuming the absence of boundary terms)

$$
\begin{aligned}
n(r) & =-\int \phi^{-1} v(r) \Xi_{0}\left[\mu-\frac{1}{2} \phi_{D}-v\right] e^{-\frac{\beta}{2} v \cdot \phi^{-1} v} D v / \Xi[\mu, \phi] \int e^{-\frac{\beta}{2} v \cdot \phi^{-1} v} D v \\
& =-\phi^{-1}\langle\langle v(r)\rangle\rangle,
\end{aligned}
$$

identifying $-\phi n(r)$ as the "mean field" $\langle\langle v(r)\rangle\rangle$. It is the approximate computation of this mean field that we must attend to, directly or indirectly.

If the field Boltzmann factor of (38) is sharply peaked about a function $\bar{v}(r)$, than of course (42) becomes simply

$$
n(r)=-\phi^{-1} \bar{v}(r)
$$

and the field $\bar{v}$ must now satisfy ( $\Omega$ is again the grand potential $-\frac{1}{\beta} \ln \Xi$ )

$$
\frac{\delta}{\delta \bar{v}(r)}\left(\Omega_{0}\left[\mu-\frac{1}{2} \phi_{D}-\bar{v}\right]\right)+\frac{1}{2} \bar{v} \cdot \phi^{-1} \bar{v}=0,
$$


or simply

$$
n_{0}\left(r \mid \mu-\frac{1}{2} \phi_{D}-\bar{v}\right)=-\phi^{-1} \bar{v}(r)
$$

the unsurprising result (comparing with $(43))$ that $n(r)$ is equal to the "bare" density $n_{0}$ in the presence of $\bar{v}$, to within an additional shift of $\frac{1}{2} \phi_{D}$. But in addition, we now have in leading order approximation the very explicit

$$
\Omega_{1}(\mu, \phi)=\Omega_{0}\left[\mu-\frac{1}{2} \phi_{D}-\bar{v}\right]+\frac{1}{2} \bar{v} \cdot \phi^{-1} \bar{v}
$$

which, since (44) represents the minimization of the kernel of (39), takes the variational form

$$
\Omega_{1}(\mu, \phi)=\operatorname{Min}_{v} \Omega_{0}\left[\mu-\frac{1}{2} \phi_{D}-v\right]+\frac{1}{2} v \cdot \phi^{-1} v .
$$

Furthermore, consistency is established by noting that by virtue of (47), $\Omega_{1}$ of (46) implies

$$
n(r)=-\frac{\delta \Omega_{1}}{\delta \mu(r)}=-\phi^{-1} \bar{v}(r)
$$

reproducing (43).

To apply (47) to the single homopolymer under discussion, we need only insert (29), serving as $\Omega_{0}$, into (47). We then have

$$
\beta \Omega_{1}[\mu, \phi]=\operatorname{Min}_{v, \psi}\left[\frac{\beta}{2} v \cdot \phi^{-1} v-2 \ln \langle 1 \mid \psi\rangle+\ln \left\langle\psi\left|e^{-\beta\left(\mu^{\prime}-v\right)}-w\right| \psi\right\rangle\right]
$$

where $\mu^{\prime}(r)=\mu(r)-\frac{1}{2} \phi_{D}(r)$. Eq. (49) can be simplified by finding and eliminating the field $\psi$, and replacing $v$ by $-\phi n$. To do so we observe that

$$
0=\frac{\delta \Omega_{1}}{\delta v}=\phi^{-1} v+e^{-\beta\left(\mu^{\prime}-v\right)} \psi^{2} /\left\langle\psi\left|e^{-\beta\left(\mu^{\prime}-v\right)}-w\right| \psi\right\rangle
$$

Integrating over the implicit r, with $N=\int n(r) d r$, then

$$
N=\frac{\left\langle\psi\left|e^{-\beta\left(\mu^{\prime}-v\right)}\right| \psi\right\rangle}{\left\langle\psi\left|e^{-\beta\left(\mu^{\prime}-v\right)}-w\right| \psi\right\rangle},
$$

reducing (50) to

$$
n=N e^{-\beta\left(\mu^{\prime}-v\right)} \psi^{2} /\left\langle\psi\left|e^{-\beta\left(\mu^{\prime}-v\right)}\right| \psi\right\rangle
$$

Since (52) is homogeneous of degree 0 in $\psi$, we are free to adopt the normalization

$$
\left\langle\psi\left|e^{-\beta\left(\mu^{\prime}-v\right)}\right| \psi\right\rangle=N
$$

converting (52) to

$$
n=e^{-\beta\left(\mu^{\prime}-v\right)} \psi^{2}
$$


(leading back to (53)), and consequently replace (49) by the simple

$$
\beta \Omega_{1}[\mu, \phi]=\operatorname{Min}_{n}\left[\frac{\beta}{2} n \cdot \phi n-2 \ln \left\langle 1 \mid n^{1 / 2} e^{\frac{\beta}{2}\left(\mu^{\prime}+\phi \cdot n\right)}\right\rangle+\ln \left\langle n^{1 / 2}\left|\left(I-e^{\frac{\beta}{2}\left(\mu^{\prime}+\phi \cdot n\right)} w e^{\frac{\beta}{2}\left(\mu^{\prime}+\phi \cdot n\right)}\right)\right| n^{1 / 2}\right\rangle\right] .
$$

appearing as a $\mu$-dependent density functional, reminiscent of "statistical models" [10-12], of polymers. Eq.(55) is our main result, valid in the mean field level. The role of the interaction $-\phi$ is transparent: the external potential is augmented by the mean field : $\mu \rightarrow \mu^{\prime}+\phi n$, and the explicit energetic component $-\frac{1}{2} n \cdot \phi n$ subtracted out. Eq.(55) is of course a density functional representation with the density profile dependence on $\mu$ determined by dropping the implicit $\phi$ and making $\mu$ explicit, by

$$
\delta \Omega_{1}[\mu, n] / \delta n(r)=0 .
$$

It is an extension of the more common form in which one is given a free energy $F[n]$ in terms of which $\mu=\delta F(n) / \delta n$, from which it follows that

$$
\delta \Omega(\mu, n) / \delta n=0
$$

where $\Omega[\mu, n]=F[n]-\mu \cdot n$. A corresponding format is obtainable here as well, but at the cost of increased complexity.

\section{NUMERICAL IMPLEMENTATION}

To exemplify the usefulness of the mean field minimization strategy, we have developed a numerical implementation of the minimization principle (55).

From preliminary simulation studies we had noticed how in the absence of a repulsive core interaction between monomers we could not converge to a stationary density. Instead the density, and therefore the total particle number, either kept on growing or shrank to zero, depending on the value of the external chemical potential. This numerical instability occures as a result of the closeness to the resonance. In these simulations we noticed, on the other hand, that despite the fact that the density itself kept changing, the global shape of density profile reached a steady state. We therefore decided to study the normalized density $p(r)=n(r) / N$. Once we had formulated the problem consistently in terms of $p$ all the numerical instabilites desappeared and we were able to determine the corresponding stady state densities and total particle numbers. 
In terms of $p$ the minimization functional (55) becomes

$\Omega_{1}[\mu, \phi]=\operatorname{Min}_{p}\left[\frac{N^{2}}{2} p \cdot \phi p-2 \ln \left\langle 1 \mid p^{1 / 2} e^{\frac{\beta}{2}\left(\mu^{\prime}+N \phi \psi\right)}\right\rangle+\ln \left\langle p^{1 / 2}\left|\left(I-e^{\frac{\beta}{2}\left(\mu^{\prime}+N \phi \psi\right)} w e^{\frac{\beta}{2}\left(\mu^{\prime}+N \phi \psi\right)}\right)\right| p^{1 / 2}\right\rangle\right]$.

Once we have determined $p(r)$, the expression to compute the particle number and the true density are

$$
\begin{aligned}
n(r) & =\frac{p(r)}{\left\langle p(r)^{1 / 2}[I-\zeta w \zeta] p\left(r^{\prime}\right)^{1 / 2}\right\rangle} \\
N & =\left\langle p(r)^{1 / 2}[I-\zeta w \zeta] p\left(r^{\prime}\right)^{1 / 2}\right\rangle^{-1}
\end{aligned}
$$

The minimization is done using a Metropolis Monte Carlo algorithm at fixed temperature, starting from a suitable ansatz for the functional form of $p(r)$. We can freely impose the form of the next neighbor potential, and therefore $w\left(r, r^{\prime}\right)$, the external confining potential $u(r)$, and the long range interaction $\phi\left(r, r^{\prime}\right)$. The main numerical difficulty arises from the need of evaluating a functional involving 6-dimensional integrals nesting 3-dimensional integrals, such as

$$
\int \sqrt{p(\mathbf{r})} e^{\frac{\beta}{2}\left(\mu^{\prime}(\mathbf{r})+N \int \phi\left(\mathbf{r}, \mathbf{r}^{\prime \prime}\right) p\left(\mathbf{r}^{\prime \prime}\right) d \mathbf{r}^{\prime \prime}\right)} w\left(\mathbf{r}, \mathbf{r}^{\prime}\right) e^{\frac{\beta}{2}\left(\mu^{\prime}\left(\mathbf{r}^{\prime}\right)+N \int \phi\left(\mathbf{r}^{\prime}, \mathbf{r}^{\prime \prime}\right) p\left(\mathbf{r}^{\prime \prime}\right) d \mathbf{r}^{\prime \prime}\right)} \sqrt{p\left(\mathbf{r}^{\prime}\right)} d \mathbf{r} d \mathbf{r}^{\prime}
$$

It turns out it is prohibitive to try to evaluate these terms directly by the use of a simple grid. Already when using 10 discretization points simulations are too slow, while the precision is very poor. To achieve higher accuracy in the integration, we use a Legendre-Gauss quadrature method with either 6 or 8 points in each linear direction.

As a test system we have chosen a square well external potential with $\mu=-\mu_{0}$ inside; a harmonic n.n. potential, leading to $w\left(\mathbf{r}, \mathbf{r}^{\prime}\right)=\exp \left(-C_{h}\left(\left|\mathbf{r}-\mathbf{r}^{\prime}\right|-\rho_{1}\right)^{2}\right)$; a long-range step potential $\phi=C_{l r} \Theta\left(x-\rho_{2}\right)$, where $\mu_{0}, C_{h}, C_{l r}$ are energy constants, and $\rho_{1}$ and $\rho_{2}$ are characteristic lengths. In the following we take the system size to be $L=1, C_{h}=5$, and $\rho_{1}=0.2$. As ansatz for the probability density we take the composition of three Gaussian in the three spatial directions, for a total of 6 parameters: $B_{x}, B_{y}, B_{z}, X_{0}, Y_{0}$ and $Z_{0}$.

$$
p(x, y, z)=A e^{-B_{x}\left(x-X_{0}\right)^{2}} e^{-B_{y}\left(y-Y_{0}\right)^{2}} e^{-B_{z}\left(z-Z_{0}\right)^{2}}
$$

At each iteration of the algorithm the normalization of $n$ is verified so that $\int p(x, y, z) d x d y d z=1$, and the value of $\mathrm{A}$ is changed accordingly.

We first look at the reference system, i.e. zero long-range interaction, figure 1. As expected the probability density converges to a single Gaussian centered in the middle of the box, with $X_{0} \sim Y_{0} \sim Z_{0} \sim L / 2$ and $B_{x} \sim B_{y} \sim B_{z}$. When the attractive potential $\phi$ is turned on the Gaussian becomes more peaked (figure 2). ). 

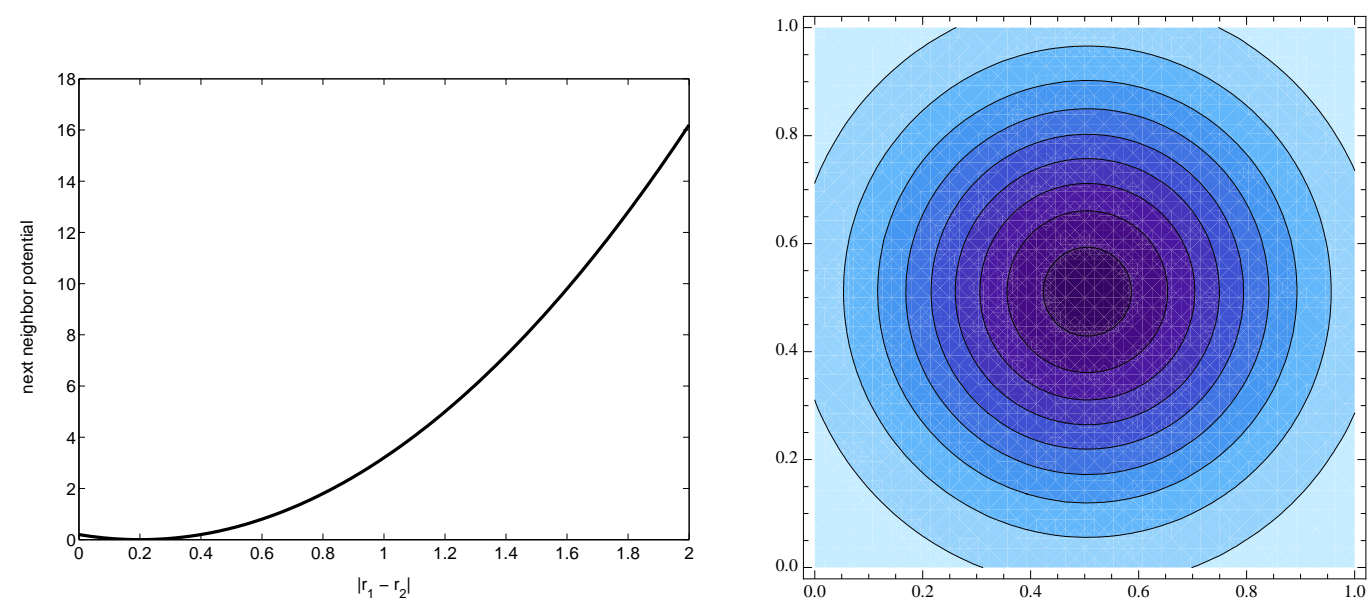

FIG. 1: Left: Interaction between neighboring particles along the chain: harmonic potential only. Right: Slice of the probability density profile taken at $z=L / 2$.
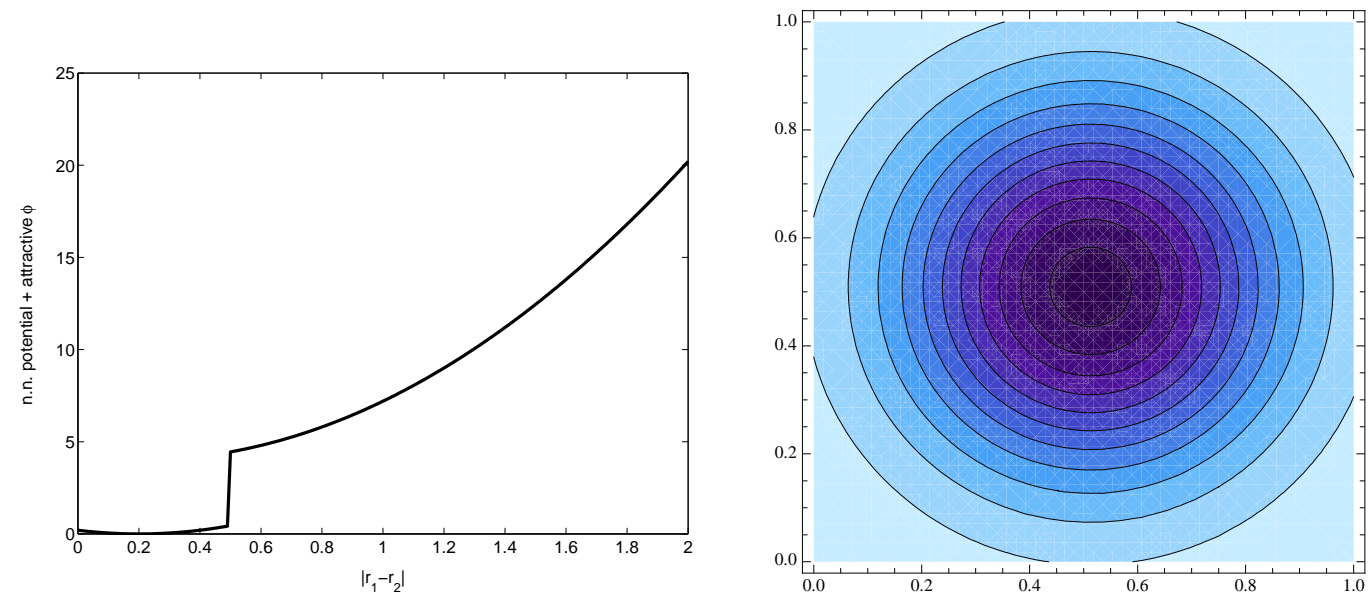

FIG. 2: Left: Interaction between neighboring particles along the chain: harmonic potential, attractive long-range interaction $\left(C_{l}=4\right), \rho_{2}=0.5$. Right: Slice of the probability density profile taken at $z=L / 2$.

Next, we introduced an asymmetry in the external potential, like a gravitational potential along $z: \mu=\mu_{0}-g z$. As expected the minimization of the functional converges to a probability density off-centered with respect to $z$ and centered in the middle of the box with respect to $x$ and $y$ (figure $3)$.

Even if the theoretical derivation of eq.(55) is valid only for strictly positive definite $\phi$, in the algorithm we can invert the sign of the long range interaction and make it repulsive, as well as attractive. We tried to go beyond the known validity of our approximation, taking a repulsive long-range interaction. Our method still holds, provided we consider repulsive interactions which 


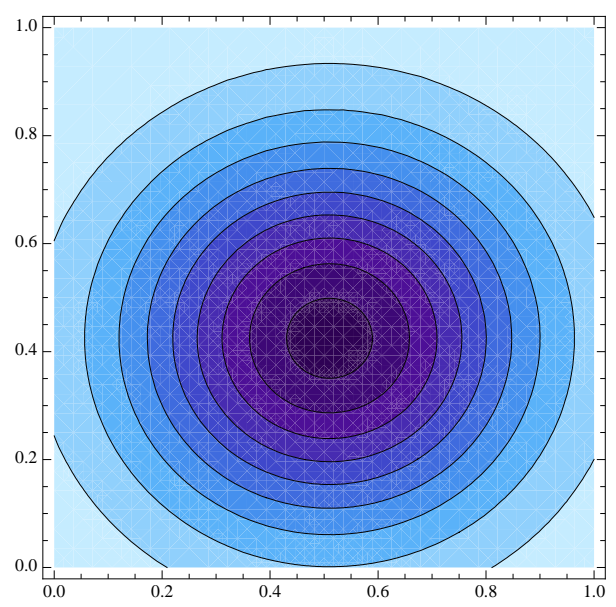

FIG. 3: Probability density profile at $z=L / 2$ in the presence of an external graviational field $(g=1$, $\left.C_{l}=2\right)$
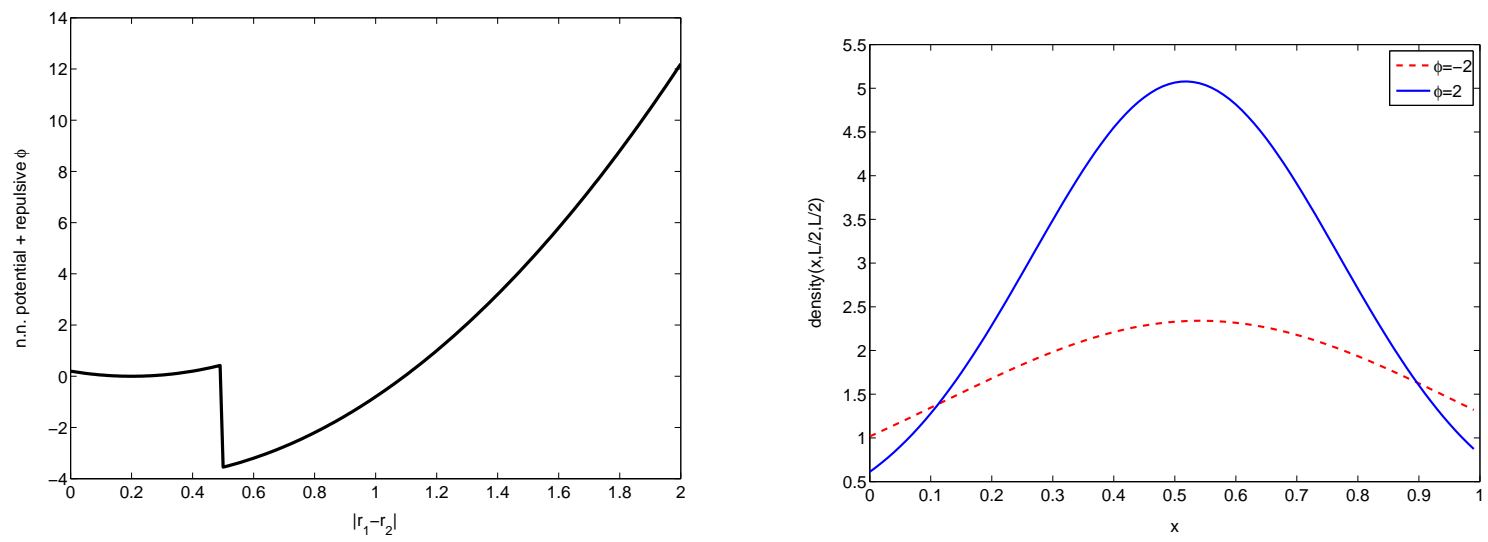

FIG. 4: Left: Interaction between neighboring particles along the chain: harmonic potential and repulsive long-range interaction $\left(C_{l}=-2\right)$. Right: probability profiles at $y=z=L / 2$ with (red) and without (blue) the repulsive core.

are not too strong. As expected a repulsive $\phi$ gives a less peaked density compared to the reference system (figure 4).

\section{LIMIT OF TIGHT CONFINEMENT}

We have seen, in Sec. II B, that in the absence of non-neighbor interactions, the large $N$ monomer distribution is determined by the "resonance state" $\psi_{\lambda_{0}}$ satisfying

$$
\left(\zeta^{1 / 2} w \zeta^{1 / 2}\right) \psi_{\lambda_{0}}=\lambda_{0} \psi_{\lambda_{0}}
$$


where $\lambda_{M A X}=\lambda_{0} \sim 1$. Explicitely, since

$$
\begin{aligned}
\left\langle r\left|\left(\zeta^{-1}-w\right)^{-1}\right| 1\right\rangle & =\left\langle r\left|\zeta^{1 / 2}\left(I-\zeta^{1 / 2} w \zeta^{1 / 2}\right)^{-1} \zeta^{1 / 2}\right| 1\right\rangle \\
& \sim \frac{1}{1-\lambda_{0}}\left\langle r \mid \zeta^{1 / 2} \psi_{\lambda_{0}}\right\rangle\left\langle\psi_{\lambda_{0}}\left|\zeta^{1 / 2}\right| 1\right\rangle
\end{aligned}
$$

we have under these circumstances, from (9)

$$
n(r) \sim \frac{1}{1-\lambda_{0}} \psi_{\lambda_{0}}(r)^{2}
$$

or since $\psi_{\lambda_{0}}(r) \sim \psi_{1}(r)$

$$
n(r) \sim \frac{\psi(r)^{2}}{1-\lambda_{0}}=N \psi_{1}(r)^{2}
$$

In other words, increasing the chain length increases the mean density uniformly, as if the floppy chain is simply winding around more under the same confinement. This uninteresting behavior is refined by two interactions that have been ignored in getting (66). First is the stiffness of successive pair orientations, equivalent to the monomers having coupled orientational degrees of freedom, a topic that has been addressed to some extent in this format in the past and will be attended to more forcefully in the future. Second is the effect of non-next neighbor interactions, which we have here studied in a preliminary fashion. The mean field $v(r)$ that has been enountered will also have the effect of correlating pair orientations, and will of course alter the nature of the long chain resonant state. Let us see how this works. To keep the extrapolation from (63-66) transparent, let us rewrite (49) (using $\zeta=e^{\beta \mu^{\prime}}$ ) as,

$$
\begin{aligned}
\Omega_{1}(\zeta, \phi) & =\operatorname{Min}_{v, \psi}\left[\frac{1}{2} v \cdot \phi^{-1} v-2 \ln \left\langle\left(\rho e^{-\beta v}\right)^{1 / 2} \mid\left(\rho^{-1} e^{\beta v}\right)^{1 / 2} \psi\right\rangle\right. \\
& \left.+\ln \left\langle\left(\rho^{-1} e^{\beta v}\right)^{1 / 2} \psi\left|I-\left(\rho e^{-\beta v}\right)^{1 / 2} w\left(\rho e^{-\beta v}\right)^{1 / 2}\right|\left(\rho^{-1} e^{\beta v}\right)^{1 / 2} \psi\right\rangle\right] \\
& =\operatorname{Min}_{v, \bar{\psi}}\left[\frac{1}{2 v \cdot \phi^{-1}-2 \ln \left\langle\left(\rho e^{-b v}\right)^{1 / 2} \mid \bar{\psi}\right\rangle+\ln \left\langle\bar{\psi}\left|I-\left(\rho e^{-b v}\right)^{1 / 2} w\left(\rho e^{-\beta v}\right)^{1 / 2}\right| \bar{\psi}\right\rangle}\right]
\end{aligned}
$$

Hence, according to (53), $\bar{\psi}=\left(\zeta^{-1} e^{\beta v}\right)^{1 / 2} \psi$ is normalized, $\langle\bar{\psi} \mid \bar{\psi}\rangle=1$ and from (54),

$$
n=N \bar{\psi}^{2}
$$

Near resonance is now signaled by the approximate validity of

$$
\left(I-\left(\zeta e^{-\beta v}\right)^{1 / 2} w\left(\zeta e^{-\beta v}\right)^{1 / 2}\right) \bar{\psi}=\bar{\lambda} \bar{\psi}
$$

with small $\bar{\lambda}>0$. Eq. (50) then tells us at once that using the exact

$$
\phi^{-1} v=-n
$$


we have

$$
N=1 / \bar{\lambda}
$$

There are two ways of making use of the approximation 69. Most directly, we substitute (68-71) into (67), obtaining

$$
\beta \Omega_{1} \sim \operatorname{Min}_{n}\left[\frac{1}{2} n \cdot \phi n-2 \ln \left\langle\left(\zeta e^{\beta \phi n}\right)^{1 / 2} \mid n^{1 / 2}\right\rangle\right]
$$

This is obviously too sweeping an approximation: by using what is effectively a first order correction in the argument of a variational principle, the role of the next-neighbor $w$ in $\beta \Omega_{1}$ has vanished. But we can pick up the next order by working instead at the "profile equation" level. It is only necessary to substitute $(68,70,71)$ directly into $(69)$ to rewrite the latter as

$$
\left(\zeta e^{\beta v}\right)^{1 / 2} w\left(\zeta e^{-\beta v}\right)^{1 / 2} n^{1 / 2}=\left(1-\frac{1}{N}\right) n^{1 / 2},
$$

and hence as

$$
\begin{aligned}
e^{-\frac{\beta}{2}(u-\phi n)} w e^{\frac{\beta}{2}(u-\phi n)} n^{1 / 2} & =\left(1-\frac{1}{N}\right) e^{-\beta \mu_{0}^{\prime}} n^{1 / 2} \\
& \equiv \Lambda n^{1 / 2}
\end{aligned}
$$

( $\mu_{0}^{\prime}$ is the global chemical potential). The operator in $(74)\left(x^{2}\right)$ has a positive kernel, hence by Jentchke's extension of Perron-Frobenius [13], the positive eigenfunction is unique (up to a multiplicative constant) and $\Lambda$ is real and maximal. This leads to what is in principle a simple numerical iteration: start eg. with $\phi=0$, compute the eigenfunction $n^{1 / 2}$ and normalize it to get $\int n(r) d r=N$. At the $k^{t h}$ stage of the M-fold iteration, replace $\phi$ by $(k / M) \phi$ and repeat the process using the current function $n$. An alternative strategy is to parameterize $n$ and determine the parameters by Galerkin, i.e. integrate (74) with weight function and solve the resulting algebraic equation.

\section{CONCLUDING REMARKS}

In this inital study, we have made one major approximation and several simplifying restrictions. The approximation is of course that of mean field, or selective neglect of fluctuations. On the assumption that fluctuations are Gaussian to leading order (examples in which this is not the case are far from rare, see e.g. [14]), a correction sequence is in primciple routine: we expand $\ln \Xi_{0}$ in 
(37) and (42) around $\bar{v}$ of (44). Using $\delta^{2} \Omega_{0} / \delta v(r) \delta v\left(r^{\prime}\right)=\delta n_{0}(r) /\left.\delta v\left(r^{\prime}\right)\right|_{\bar{v}}$,

$$
\begin{aligned}
& \Xi_{0}\left[\mu-\frac{1}{2} \phi_{D}-v\right] \exp \left\{-\frac{1}{2} \beta v \cdot \phi^{-1} v\right\}= \\
& =\exp \left\{-\beta\left(\Omega_{0}\left[\mu-\frac{1}{2} \phi_{D}-\bar{v}\right]+\frac{1}{2} \bar{v} \cdot \phi^{-1} \bar{v}+\frac{1}{2}(v-\bar{v}) \cdot\left[\left.\frac{\delta n_{0}}{\delta v}\right|_{\bar{v}}+\phi^{-1}\right](v-\bar{v})+\ldots\right)\right\}
\end{aligned}
$$

It readily follows (see e.g. [15]) that the density profile is given to the next order by simply averaging the density over the Gaussian field fluctuation:

$$
n(r)=\frac{\int n_{0}\left[\mu-\frac{1}{2} \phi_{D}-\bar{v}-\Delta\right] e^{-\frac{1}{2}\left(\frac{\Delta^{2}}{M^{-1}(r, r)}\right)} d \Delta}{\int e^{-\frac{1}{2}\left(\frac{\Delta^{2}}{M^{-1}(r, r)}\right)} d \Delta}
$$

where $M\left(r, r^{\prime}\right)=\phi^{-1}\left(r, r^{\prime}\right)-n_{0}^{\prime}\left[\mu-\frac{1}{2} \phi_{D}-\bar{v}-\Delta\right]$; here, $\Delta$ is the field amplitude fluctuation at $r$. The simplifying assumptions attend more directly to the physics, and these assumptions depend very much on the nature of the system to be studied. Taking these assumptions in order, we first emebedded out system in a monomer grand ensemble. Since preprocessing assures that in practice one does not deal with the resulting extreme polydispersity, a fixed $N$ ensemble is more relevant than fixed $\zeta$. The corresponding inverse mapping has been attended to on numerous occasions (see e.g. [16]). The same formalism is indeed avaliable here (hinted at in eqs.(24),(25)).

Another restriction was to attractive long-range forces. We found however that the profile equation could indeed be pushed into the partially repulsive regime, although the validity of the minimum principle was in question; this is closely related to the functional Fourier transform for the repulsive component, likewise under unceirtain control. An alternative approach lies in the use of the mean spherical model $[17,18]$ and its extensions. This is the aim of ongoing research.

Of course, there is the implicit assumption that pair forces suffice, whereas the action of pairs on singlets is a frequent important observation, leading e.g. to dihedral angle dependence in chains. Typically (see e.g. [19] for a very primitive example) one can simply create a multi-unit monomer to encompass only such forces, which then appear once more as pair forces.

Most blatantly, our approach has been restricted to homopolymers. Since the set of degrees of freedom of a monomer can include monomer type, this is no restriction at all if one is studying the effect on the full population of an assumed relative frequency of occurrence of next neightbors hetero-pairs. However, if we attribute a sense to the chain and the AB frequency differs from the BA frequency, the very convenient symmetry of the operator $w$ is lost, and with it, the possibility e.g. of a specific long sequence of monomer types. The case of non-symmetric $w$ has indeed been studied [20], but exercising the kind of control that we have in our current formulation remains a 
challenge.

[1] P.G. de Gennes, "Scaling concepts in polymer physics", Cornell University Press (1979)

[2] I.M. Lifshits, Sov. Phys. JETP 55, 2408 (1968)

[3] S. Pasquali and J.K. Percus, J. Chem. Phys. 125, 064906 (2006)

[4] H.L. Frisch and J.K. Percus, J. Phys. Chem. B 105, 11834 (2001)

[5] J.K. Percus, J. Stat. Phys. 106, 357 (2002)

[6] M. Kac, in "Applied Probability", ed C.A. MacColl, McGraw Hill, NY (1957)

[7] A.J.F. Siegert, Physica (supp.) 26, 530 (1960)

[8] J. Hubbard, Phys. Rev. Lett. 3, 11 (1959)

[9] R.L. Stratonovich, Sov. Phys. Doklady 2, 416 (1958)

[10] G. Giacomin, "Random Polymer Models", World Scientific (2007)

[11] P. Flory, "Statistical mechanics of chain molecules", Wiley (1969)

[12] M. Huggins, J. Phys. Chem. 46, 151 (1942)

[13] R. Jentzsch, Crelles Jour. 141, 235 (1912)

[14] R. Rajaraman, Phys. Rep. C 21, 227 (1975)

[15] J.K. Percus, Physica A 172, 1 (1991)

[16] J.L. Lebowitz, J.K. Percus, and L. Verlet, Phys. Rev. 153, 2506 (1967)

[17] J.K. Percus and G.J. Yevick, Phys. Rev. 136, 290 (1964)

[18] J.L. Lebowitz and J.K. Percus, Phys. Rev. 144, 251 (1966)

[19] K.K. Muller-Nedebock, H.L. Frisch and J.K. Percus, Phys. Rev. E 67, 011801 (2003)

[20] H.L. Frisch and J.K. Percus, Phys. Rev. E 64, 011805 (2001) 